\title{
A case of acquired urachal pathology in a child
}

\author{
N. Dilwali ${ }^{1}$, C. Persaud ${ }^{2}$, J. Gallucci ${ }^{3}$ \\ ${ }^{1}$ Rutgers Robert Wood Johnson Medical School, USA \\ ${ }^{2}$ St. Joseph's Regional Medical Center, USA \\ ${ }^{3}$ Saint Peter's University Hospital, USA
}

Key words: Urachal cyst; patent urachal sinus;

laparoscopy; umbilical trocar; infected urachal cyst

\section{Introduction}

The urachus is a fibrous embryological remnant of the allantois, a narrow canal structure that connects the top of the foetal bladder to the umbilicus (1). This canal is initially patent but later closes as the bladder descends into the pelvis and stretches the urachus. The median umbilical ligament is the resultant adult remnant, which runs from the umbilicus to the dome of the bladder. Urachal pathology can present as congenital or acquired. Failure of the urachus to close leads to congenital urachal defects including urachal cyst, urachal sinus, patent urachus and vesicourachal diverticulum. Urachal anomalies usually occur as isolated defects and are typically not associated with syndromes except prune belly syndrome.

In the literature and clinically, urachal sinus and cyst are more common than a patent urachus and vesicourachal diverticulum (2). 1 in 5,000 live births lead to a urachal cyst, but only 1 in 150,000 become clinically relevant in the population(1).

The most common signs of a patent urachus are clear drainage of urine through the umbilicus, abnormal appearing umbilicus or infectious findings; these commonly are seen in the neonatal period, most other anomalies are asymptomatic unless complicated by an infection (3). Routes of infection include haematogenous and direct spread from the bladder or the lymphatic system. Ultrasound is appropriate for confirming a diagnosis of urachal pathology, computed tomography (CT) scan and magnetic resonance imaging (MRI) are alternative imaging techniques. In infants, ultrasonography has up to 100 percent sensitivity when identifying a patent urachus, 100 percent sensitivity for urachal sinus and 82 percent sensitivity in identifying urachal cysts (4). Treatment of urachal pathology is the removal of the complete structure to prevent malignant transformation in

Correspondence: Natasha Dilwali

E-mail: ndilwali@gmail.com

Received: 11-03-2019 Accepted: 18-04-2019

(iD) http://orcid.org/0000-0001-5557-3744

DOI: http://doi.org/10.4038/sljs.v37i1.8603

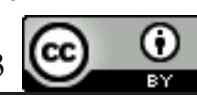

adulthood to urachal carcinoma. In our review, there have been no paediatric cases reported of urachal pathology due to instrumentation from a previous laparoscopic procedure.

\section{Presentation}

An eight-year-old male with a history of a laparoscopic appendectomy two years prior presented for resection of an infected patent urachus. The patient was born full-term via Caesarean section with no complications at birth nor in the first year of life. The patient's family reported no discharge from the umbilicus until three months before the elective surgery; the patient had developed cellulitis of his periumbilical area, presented to a nearby emergency room and was prescribed a ten-day course of topical antibiotics. During these ten days, the patient developed umbilical discharge that went from purulent to clear. Following these episodes, the family presented to their paediatrician who attempted to cauterize the umbilicus.

However, the drainage recurred soon after prompting surgical evaluation. Upon ultrasound and CT scan imaging, a patent urachal cyst and sinus were confirmed. The patient underwent an uncomplicated excision of a patent urachus with bladder cuff and urachal tract with placement of Penrose drain through the congenital urachal sinus at the umbilicus. Intraoperative wound cultures grew Enterococcus coli; the patient received three days of intravenous Piperacillin/Tazobactam and was discharged on oral Cephalexin for seven days.

\section{Discussion}

Our patient presented for surgical removal of an infected patent urachus two years after undergoing a laparoscopic appendectomy with umbilical, right upper quadrant and left lower quadrant ports. During the appendectomy and its preoperative imaging, there was no indication of urachal pathology.

The patient's previous laparoscopic procedure, utilizing an umbilical port, could be a cause of the acquired urachal pathology requiring operative intervention. Prior to the patient's appendectomy, there was no history of umbilical pathology. It is possible the patient had an asymptomatic patent urachus that was missed on preoperative imaging, and during the appendectomy, however, it is more likely the 
urachal pathology was acquired after the laparoscopic procedure as the patient began to demonstrate symptoms after the appendectomy with imaging confirming its presence. When performing laparoscopic procedures in the paediatric population, we must be aware of the possibility of acquired urachal pathology due to trocar placement.

All authors disclose no conflict of interest. The study was conducted in accordance with the ethical standards of the relevant institutional or national ethics committee and the Helsinki Declaration of 1975 , as revised in 2000 .

\section{References}

1. Gami BL, Biswas S. An infected urachal cyst. BMJ Case Rep. 2013 Feb 21; 2013:1-2. doi:10.1136/bcr-2012-007105

2. Gearhart JP, Rink RC, Mouriquand PDE. Pediatric Urology. 2nd ed. Philadelphia, PA: Sauders/Elesevier; 2010.

3. Naiditch JA, Radhakrishnan J, Chin AC. Current diagnosis and management of urachal remnants. J Pediatr Surg. 2013 Oct; 48(10):2148-52. doi:10.1016/j.jpedsurg.2013.02.069

4. Yiee JH, Garcia N, Baker LA, Barber R, Snodgrass WT, Wilcox DT. A diagnostic algorithm for urachal anomalies. J Pediatr Urol. 2007 Dec; 3(6): 500-4. doi:10.1016/j.jpurol.2007.07.010

\section{Learning Points:}

- Most urachal anomalies are asymptomatic unless complicated by an infection. Common signs of a patent urachus are clear drainage of urine through the umbilicus or abnormal appearing umbilicus.

- Urachal pathology can present as congenital or acquired; most are seen as congenital forms due to the failure of closure of the urachus causing a urachal cyst, urachal sinus, patent urachus, and vesicourachal diverticulum.

- During laparoscopic procedures in the paediatric population, we must be cautious of the possibility of acquired urachal pathology due to trocar placement. 